ఠ

\title{
Nanosilica-supported liposome (protocells) as a drug vehicle for cancer therapy
}

This article was published in the following Dove Press journal:

International Journal of Nanomedicine

\author{
Vinay K Belwal' \\ KP Singh ${ }^{1,2}$
}

'Bio-Nanotechnology Research Laboratory, Biophysics Unit, CBSH, GB Pant University Agriculture and Technology, Pantnagar, Uttarakhand, ${ }^{2}$ Department of Molecular Biology, Biotechnology \& Bioinformatics, $\mathrm{COBSH}, \mathrm{CCS}$ Haryana Agriculture University, Hisar, Haryana, India
Correspondence: KP Singh

CCS Haryana Agriculture University,

Hisar 125004, Haryana, India

Tel +9l 94 I2632572

Fax +9| 05944230567

Email kps_biophysics@yahoo.co.in

\begin{abstract}
This study encompasses the development and comparison of nanosilica-supported liposome (protocells), conventional liposome, and polyethylene glycol (PEG)-liposome. An effort was made to study the drug encapsulation efficiency and the in vitro release of the drug, and whether protocells (nanovesicles) could sustain the release of the drug by increasing the residence time, which could reduce the dose-related systemic toxicity of the drug, that is, vincristine sulfate. Nanovesicles had a good encapsulation efficiency (71\%), which was comparable to the conventional and PEG-liposome, which were $74 \%$ and $78 \%$, respectively. The obtained vesicles were in the size range $100-150 \mathrm{~nm}$, and the drug release efficiency of conventional, PEGylated, and protocells liposome was about $67 \%, 42 \%$, and $52 \%$, respectively, in 150 minutes. The intermediate value of nanosilica-supported liposome indicates the ability for stable and controlled release of the drug, which prevents the rapid burst or slower release of the drug. This study reveals that protocells as nanovesicles could be a better choice for the delivery of cancer drugs such as vincristine sulfate.
\end{abstract}

Keywords: mesoporous silica nanoparticles, nanovesicles, protocells, nanodimensions, nanosilica supported liposomes

\section{Introduction}

Cancer is the leading cause of death in economically developed countries and the second leading cause of death in developing countries. Cancer chemotherapy is generally accompanied by side effects. If an anticancer drug could deliver only the right site in the right concentration at the right time, cancer could be cured without side effects. One of the main goals of nanomedicine is to develop a nanocarrier that can selectively deliver anticancer drugs to target tumors and affect as few healthy cells as possible. Several different types of drug carriers or "vehicles" have been developed for this task, including polymers, liposomes, dendrimers, and inorganic nanoparticles. ${ }^{1}$ Utilizing these vehicles has several advantages over conventional dosage methods, including decreased drug inactivation, increased efficacy, and reduced nonspecific interactions. Recently, one type of inorganic nanoparticle that has been successfully applied as a controlled-release drug delivery system is mesoporous silica nanoparticle materials. ${ }^{2}$ Amorphous mesoporous silica particles were suggested as useful carriers because of their stability, controllable pore diameter, and biocompatibility. ${ }^{3}$ Therefore, in continuation of our previous work, ${ }^{4,5}$ we prepared and characterized vincristine sulfate-encapsulated nanosilica-supported liposome (protocells) as nanovesicles and compared it with conventional and polyethylene glycol (PEG)-coated or stealth liposomes to evaluate the encapsulation efficiency and in vitro performance (Figure 1). 

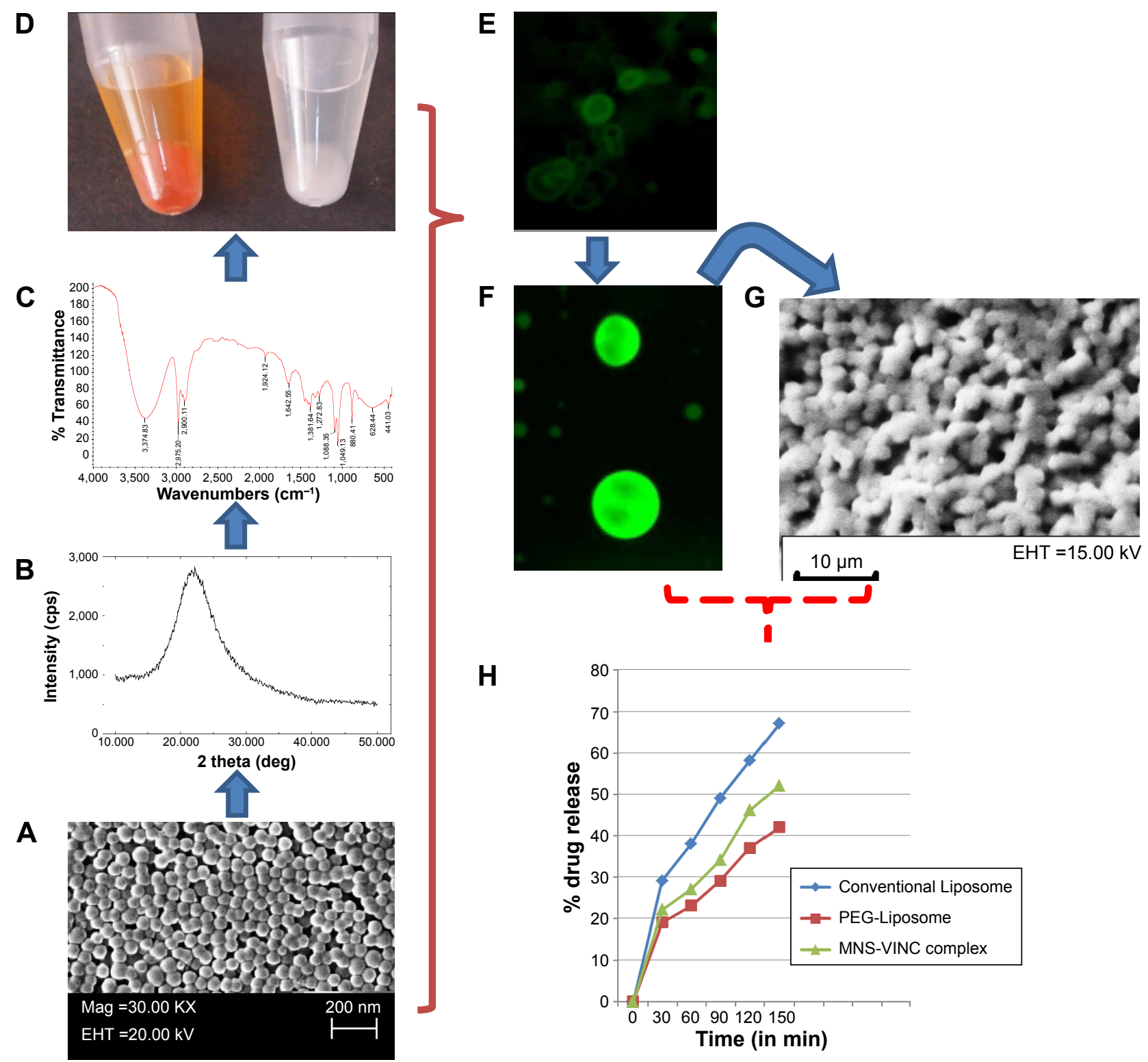

H

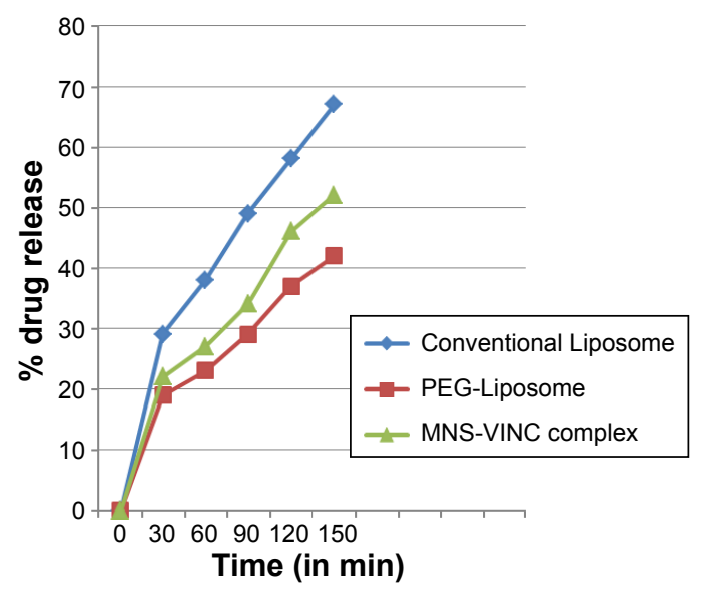

Figure I (A) Scanning electron micrograph (SEM) of MNS; (B) XRD crystallography of MNS; (C) FTIR spectra of MNS; (D) MNS loaded with dye FITC (orange) and vincristine sulfate (white); (E) fluorescent image of MNS encapsulated in liposome. (F) Fluorescent image of MNS - FITC complex; (G) SEM images of MSN-encapsulated liposomes (protocells) and (H) in vitro drug release in conventional, PEGylated and MNS supported protocells.

Abbreviations: MNS, mesoporous nanosilica; XRD, X-ray diffraction; FTIR, fourier transform infrared spectroscopy; EHT, electron high tension; FITC, fluorescein isothiocyanate; VINC, vincristine sulfate.

\section{Materials and methods}

The conventional liposomes were prepared using a rapid evaporation method. The polyethylene glycol (PEG-2000) was attached to phosphatidylcholine (PC) by dissolving them in a ratio of PC:PEG (4:2) in $10 \mathrm{~mL}$ of chloroform (organic solution). The solution was then evaporated using rotatory evaporator at $60^{\circ} \mathrm{C}$ for 45 minutes at $200 \mathrm{rpm}$. After preparing the PC-PEG complex, the PEG liposomes were prepared by mixing PC:CH:PC-PEG in the ratio of 5:3:2 in $10 \mathrm{~mL}$ of organic solution, and dried to form a thin lipid film in rotary evaporator as explained above and same was reconstituted in HBSE buffer (10 mM HEPES, $150 \mathrm{mM}$ $\mathrm{NaCl}, 9.1 \mathrm{mM}$ EDTA; $\mathrm{pH} 7.5$ ). The solution was then incubated overnight with continuous stirring. After overnight incubation, the free drug was removed by multiple rounds of centrifugation, supernatant removal, and washing, until no or little drug was detectable in the supernatant. Preparation of FITC-mesoporous silica nanoparticle-encapsulated fluorescence liposome (protocell) was done as per the method prescribed. ${ }^{5}$ Removal of free drug, determination of drug encapsulation efficiency and drug loaded in mesoporous nanosilica, characterization of mesoporous nanosilica, 
characterization of liposome, and study of in vitro drug release from the liposomes were done by standard established methods.

\section{Results and discussion}

The drug release mechanisms of conventional liposomes, stealth liposomes, and mesoporous nanosilica-loaded drug were comparable. The release rate was highest for the drug encapsulated in the conventional liposome, while the rate of release from PEG-liposomes was less than that of conventional liposomes and mesoporous nanosilica-loaded drug. Thus, it can be concluded that by adding PEG to the liposomes, the release rate of the drug from the liposomes is decreased due to some steric hindrance. This is due to the barrier against diffusion of the hydrophilic drug, created by the hydrophobic long alkyl chains of the polymer, and the drug was effectively entrapped in the polymers. Mesoporous nanosilica shows in vitro release of the drug between conventional and stealth liposomes which show a stable in vitro release of the drug, and can be used to deliver the drug to cancer cells and tumors. The burst effect varies with the liposome type and lipid in case of liposome, and in case of mesoporous silica, it depends upon the pore size on the surface of mesoporous silica. The ability to control the release of anticancer drugs can provide mesoporous silica nanoparticles with advantages over other drug delivery systems such as PEGylated liposomal particles or conventional liposomal particles. ${ }^{6}$ On the basis of the above results, it can be suggested that the drug would be stable in the blood circulation and would be released slowly at the cancerous site. It is also evident from the above study that our PEGylated liposomal formulation and drug-loaded mesoporous nanoparticles meet the requirements for an effective drug delivery system. ${ }^{7}$

\section{Conclusion}

The results suggest that the encapsulation of vincristine sulfate and its release from the nanosilica-supported liposome occur for a prolonged period of time. The value is comparable with the PEGylated liposomal formulations. Since the PEGylated nanosilica supported protocells are of nanosize, they can load different dyes for color contrast to distinct in visualization during imaging, and also have longer residence time in body fluid under circulation which can help them to reach the targeted tissues. Nanosized silica-supported liposomes may be promising delivery systems for cancer drugs in cancer therapy. These studies are preliminary, and therefore, further studies are needed including in vivo experiments to show the suitable role of such nanoscale protocells in drug delivery.

\section{Acknowledgments}

The authors acknowledge with thanks the facilities provided by the experimental directorate, Govind Ballabh Pant University of Agriculture and Technology, Pantnagar and the Centre of Excellence for Mountain Biology, Uttarakhand Council for Biotechnology, Haldi, US Nagar, Uttarakhand, India.

\section{Disclosure}

The authors report no conflicts of interest in this work.

\section{References}

1. Trewyn BG, Giri S, Slowing II, Lin VSY. Mesoporous silica nanoparticle based controlled release, drug delivery, and biosensor systems. Chem. Commun (Camb). 2007;31:3236-3245.

2. Vivero-Escoto JL, Slowing II, Trewyn BG, Lin VSY. Mesoporous silica nanoparticles for intracellular controlled drug delivery. Small. 2010; 6(18):1952-1967.

3. Barbe C, Bartlett J, Kong L, et al. Silica particle: a novel drug delivery system. Adv Mater. 2004;16(1):1959-1966.

4. Panwar P, Pandey B, Lakhera PC, Singh KP. Preparation, characterization, and in vitro release study of albendazole encapsulated nanosize liposomes. Int J Nanomedicine. 2010;5:101-108.

5. Singh KP, Panwar P, Kohli P, Sanjesh. Liposome mesoporous silica nanoparticles fused cores: a safer mode of drug carrier. J Biomed Nanotechnol. 2011;7(1):60-62.

6. Lu J, Liong M, Zink JI, Tamanoi F. Mesoporous silica nanoparticles as a delivery system for hydrophobic anticancer drugs. Small. 2007;3(8): 1341-1346.

7. Yousefi A, Esmaeili F, Rahimian S, Atyabi S, Dinarvand R. "Preparation and in vitro evaluation of a pegylated nano-liposomal formulation containing docetaxel. Sci. Pharm. 2009;77:453-464.
International Journal of Nanomedicine

\section{Publish your work in this journal}

The International Journal of Nanomedicine is an international, peerreviewed journal focusing on the application of nanotechnology in diagnostics, therapeutics, and drug delivery systems throughout the biomedical field. This journal is indexed on PubMed Central, MedLine, CAS, SciSearch ${ }^{\circledR}$, Current Contents ${ }^{\circledR} /$ Clinical Medicine,

\section{Dovepress}

Journal Citation Reports/Science Edition, EMBase, Scopus and the Elsevier Bibliographic databases. The manuscript management system is completely online and includes a very quick and fair peer-review system, which is all easy to use. Visit http://www.dovepress.com/ testimonials.php to read real quotes from published authors. 